\title{
IMPLEMENTING ENTERPRISE VOIP DEPLOYMENT
}

\author{
Engr. Oluseyi Basorun ${ }^{1 *}$, MNSE MNIEEE MIEEE ${ }^{2}$ \\ *1Electronic and Computer Engineering department, Lagos State University, EPE \\ *Email: Oluseyiseyi2004@yahoo.com
}

*Corresponding Author: -

Email ID - Oluseyiseyi2004@yahoo.com

\begin{abstract}
: -
Voice over Internet Protocol (VoIP) is perceived as the best example of collaboration technology in today's telecommunication space. There have being a paradigm shift from regular legacy telephone systems to VoIP over the last decade by most telecommunication and multinational companies globally. One of the huge benefit of VoIP is noticeable reduction in international call rates. In addition to the potential cost savings, VoIP offers the prospect of integrating voice with data and video applications, this goes a long way in increasing workers' productivity within organizations. Many organizations are already embracing VoIP as replacement for their legacy PBX, others are integrating the existing PBX system with VoIP. This write up will focus on deployment of PBX-VoIP converged solution in enterprise organizations, where cost saving is one of the primary objectives.
\end{abstract}

Keywords: Enterprise, Deployment, collaboration technology

\section{(ㄷ) (\$) (1)}




\section{INTRODUCTION}

In a few years to come VoIP will be the next dominant technology for enterprise communication. VoIP services operate on Internet protocol (IP) to transmit compressed voice samples as frames and messages as IP packets over an IP data network. VoIP can be deployed on any IP data network. Examples are Internet, WAN, and LANs. In this discuss private organizational networks spanning over more than one geographical locations also known as Wide Area Network (WAN) was taken into consideration. Voice from telephone lines is converted into a suitable signal level, digitized, compressed as voice payload, and sent as IP packets. SIP protocols are used to set up and tear down calls between users, carry users' location information, negotiate capabilities, set up call features like user ID, call waiting and call forwarding. Due to the simplicity of architecture and numerous gains of the VoIP technology, a lot of corporate firms all over the world are taking advantage of VoIP deployment for their intranet communications, Nigeria won't be an exception.

An enterprise organisation with an installed circuit-switch PBX system has several option to migrate to IP telephony. One of the options is to introduced media gateway to link the IP equipment (IPBX and digital trunk circuit interfaces) with the legacy PBX. A T1/E1 or PRI card will interface VoIP system with the existing PSTN central office or PBX in the office. This implementation is referred to as converged IP-BX system. With the advent of internet and data connectivity, VoIP is seen as the strategic and most popular option for a cost effective enterprise telephone solution.

\subsection{VoIP vs Legacy PBX}

A table comparing the properties of VoIP service to PBX service is presented below. It can be observed that while VoIP is cost effective, flexible in deployment and with additional features as compared to legacy PBX, security and quality of service issues still require improvement. Mitigation against these known issues are discussed in subsequent sections.

\begin{tabular}{|l|l|l|}
\hline Property & VoIP Service & Legacy PBX Service \\
\hline Cost & $\begin{array}{l}\text { The main advantage of VoIP is } \\
\text { cost, both for the initial } \\
\text { investment on equipment and } \\
\text { monthly charges. Calls are free }\end{array}$ & $\begin{array}{l}\text { Substantial costs are involved to } \\
\text { purchase the equipment and for } \\
\text { monthly charges on local and } \\
\text { long distant calls }\end{array}$ \\
\hline Reliability & $\begin{array}{l}\text { A main disadvantage of a VoIP } \\
\text { system is its reliance on the } \\
\text { Internet to function. In the event } \\
\text { of power failure or outage of } \\
\text { internet service, calls across } \\
\text { geographical location will not be } \\
\text { possible }\end{array}$ & $\begin{array}{l}\text { Traditional PBX lines rely on } \\
\text { PSTN alls across geographical } \\
\text { locations }\end{array}$ \\
\hline Flexibility of provisioning & $\begin{array}{l}\text { With VoIP, you can add } \\
\text { telephone/extension lines and } \\
\text { only limited by the bandwidth of } \\
\text { your network connection }\end{array}$ & $\begin{array}{l}\text { With PBX, you buy a system } \\
\text { designed for a particular } \\
\text { numbers of extension lines. If } \\
\text { you need extra lines, you pay } \\
\text { extra for expansion cards }\end{array}$ \\
\hline Voice Quality & $\begin{array}{l}\text { Voice quality of VoIP depends } \\
\text { on the quality of internet or } \\
\text { network connections }\end{array}$ & $\begin{array}{l}\text { Voice quality is good, } \\
\text { guaranteed with 64kbps } \\
\text { bandwidth reservation }\end{array}$ \\
\hline Switching Technology & Packet Switched & Circuit Switched \\
\hline Protocol & Uses SIP, H323, RTP,H.245, IP & SS7 signal protocol \\
\hline Security & High Security threats & Minimum security threats \\
\hline
\end{tabular}

\subsection{Benefit of Enterprise VoIP Deployment}

Many organizations today are making the switch over to enterprise VoIP communication systems. The reason for the commonness of VoIP is that it gives significant benefits compared to legacy phone systems. Some of the key drive for implementing VoIP solution across organizations are enumerated below:

1. Need to save cost on Long distance calls. Most organizations spend a lot on calls costs, this can be reduced greatly as most of the charged calls can be routed through the VoIP system.

2. Cost reduction on communication equipment: Businesses can save a lot of funds through reduced upfront capital investment on communication systems. VoIP equipment hardware housed fewer port circuit cards, this makes it less expensive when compare with legacy PBX system. Integrating headquarters and remote offices into one. As calls may be hitting the central reception in headquarters it can be transferred to users down the hall or to a staff 1000 kilometers away in other remote branches

3. Create platform for video and voice solution for cross function meetings.

4. Implement Voice messaging and unified communication to assist in business processes, e.g. voice mail.

5. Roaming of staff extension profiles across office locations. Staff can work remotely from their home office and still be able to pick clients calls on their extensions.

6. Replacing or integrating with Private Branch Exchange (PBX). 
7. Increased productivity, VoIP extensions can ring on multiple devices before forwarding to voice mail.

8. Option of softphones in replacement for physical phone boxes.

9. Ongoing expenses in operation and maintenance of personnel can be reduced when combine voice and data on the same platform. There won't be need to hire separate technicians for telephony system

10. Fewer transmission resources are required when using VoIP codec

\section{DESIGN CONSIDERATIONS}

For successfully deployment of VoIP solution over enterprise organization's IT infrastructure, there are a number of critical network considerations that must be observed especially if the infrastructure spans over different locations. End users VoIP audio quality experience must be equal to public switched telephone networks (PSTNs), the backend transmission infrastructure has a lot to offer in ensuring efficient delivery of voice traffic over IP networks with little or no noticeable service degradation. Usually in traditional circuit-switched PSTNs, echo, crosstalk, voice level variation are some of the issues encountered. On the other hand packet-switched VoIP experiences, delay, jittery and packet loss. The acceptable quality of voice for a good user's experience is rated as Mean Opinion Score (MOS) of 4 on a 5 point scale. A proper network infrastructure design must be in place to mitigate against these known voice quality issues.

Bandwidth: For a jitter free voice implementation there is need to take into consideration the type of protocol to be implemented. VoIP consider three main protocols groups:

- SIP, H.323, MGCP

- The Real-Time Transport Protocol (RTP)

- ITU standard CODEC

Most implementation reserve just good enough bandwidth required for 100 concurrent calls by users. Implementing QoS and traffic engineering within LAN and WAN switching \& routing infrastructures is a key requirement for a near traditional telephone experience.

Packet loss and delay: During the design stage, packet loss must be considered, this should not be more than $1 \%$ for a good users' experience. Delay metrics must be calculated as $150 \mathrm{~ms}$ for domestic VoIP traffic and 200ms for international traffic, these are best international practices.

Audio Codec and Compression: For most VoIP solutions, ITU standard CODEC G.711 and G.729 are supported. G.711 standard digitizes voice into 64kbps before encapsulation with no compression while G.729 codec compresses voice at $8 \mathrm{kbps}, 8$ to 1 compression. This is the ideal CODEC for WAN and low bandwidth circuits.

\section{VoIP Protocols}

VoIP protocols are implemented for establishing presence, locate users, set up, modify and tear down sessions, transmission of packetized audio/video and other supporting activities like AAA and QoS. In this section we will discuss three types of protocols which are widely used in VoIP implementations. These protocols for discuss are H.323 protocols, the Session Initiation Protocol (SIP) and the media Gateway Controller Protocol (MGCP).

\section{H.323 Protocol}

H.323 is a standard approved by International Telecommunication Union (ITU) in 1996 to promote compatibility in videoconference transmissions over IP networks. It consists of groups of protocols that are used for call set-up, call termination, registration, authentication and other functions. These protocols are transported over TCP or UDP protocols stack. The H.323 family of protocol consists of H.225 for registration, admission, and call signaling. H.245 is used to establish and control the media sessions. T.120 is used for conferencing applications in which desktop and white-board application can be shared. The audio codec is defined by G.711, G.722, G.729 and G.723.1, while video codec is defined by H.261 and H.263 series of specifications. H.323 deploys RTP for media transport and RTCP is used for purpose of controlling RTP sessions.

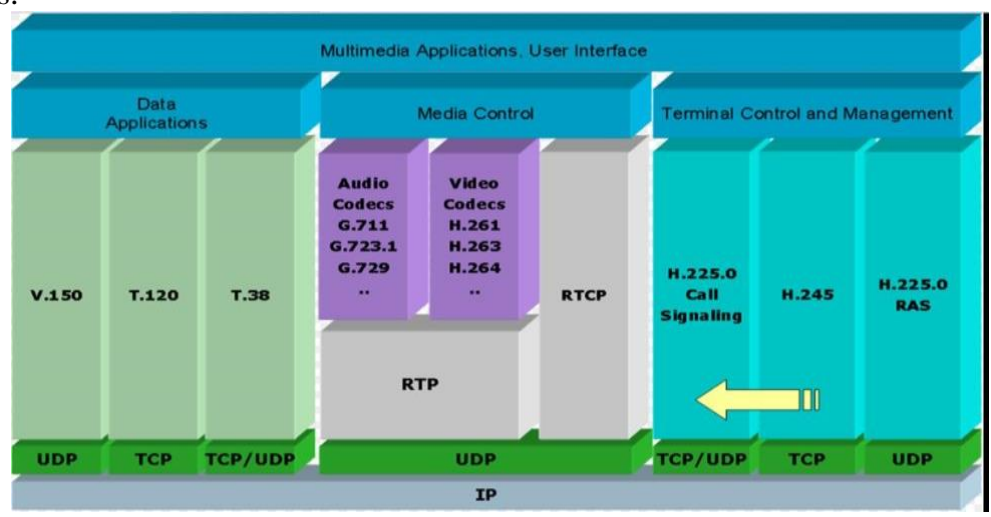

Session Initiation Protocol (SIP)

Fig 1: H.323 Protocol family [3] 
SIP is end-to-end, client-server session signalling protocol. it was developed initially in 1999 by Internet Engineering Task Force (IETF) Multiparty Multimedia Session Control Group (MMUSIC) for multimedia conferencing over IP. This was later updated by SIP WG in 2002. SIP's primarily provides presence, mobility, session setup and termination services. Other arbitrary services built on top of SIP are redirection of calls and reply with a webpage if unavailable [2]. Similar to http, SIP messages can convey arbitrary signalling payload: session description, instant messages, JPEGs, any MIME types. SIP components are following:

- SIP Proxy Server

- SIP Redirect Server

- $\quad$ SIP Registrar

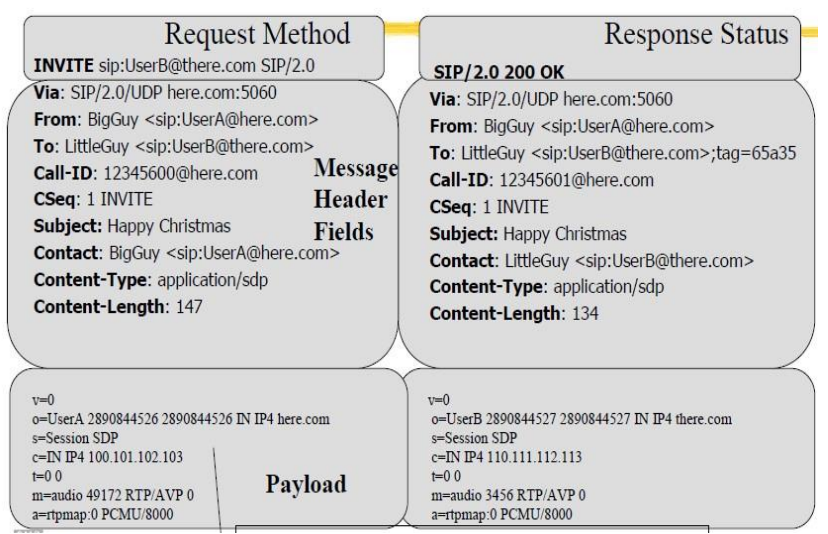

Fig 2: SIP Message structure

\section{Media Gateway Controller Protocol (MGCP)}

MGCP is a signaling and call control communications protocol used in Voice over IP (VoIP) systems. Also known as H.248, this is a standard protocol for handling the signaling and session management needed during VoIP session. The protocol defines a means of communication between a media gateway, which converts data from the format required for a circuit-switched networks (PSTN or PABX) to that required for a packet-switched network and the media gateway controller. MGCP can be used to set up, maintain, and terminate calls between multiple endpoints. Megaco and H.248 refer to an enhanced version of MGCP.

\subsection{Network Planning}

Many organizations, today, are considering converging voice and data on the same LAN and need to ensure that their existing networks can take on this additional load. In general, the transition to VoIP is a significant change to the network environment, WAN and LAN backbone capacity planning will be required in the event of multiple VoIP sites. Most data network has been optimized and honed to support the unique data applications the business requires. The WAN backbone can be an MPLS L2VPN or L3VPN or an IPLC built on dedicated ISP circuit connections. Integrating telephony services places new demands on the network resources in terms of capacity and QoS requirements. Bandwidth capacity planning requires an understanding of how many users will be at each location, likely numbers of concurrent calls, how much bandwidth each user needs etc. It is commonly known that QoS is a key factor in implementing VoIP solution, and MPLS has offered a robust platform for provider to deploy a very effective service quality. Internet data traffics are most of the time transmitted using best effort and effective transmission of traffics cannot be guaranteed on best effort. For an acceptable voice transmission over the internet, implementing QoS is very important and this should be considered in the design phase of the deployment.

Fig. 1 below is an architectural design of VoIP over WAN. The design integrates existing legacy Panasonic PBX devices into an enterprise VoIP system.

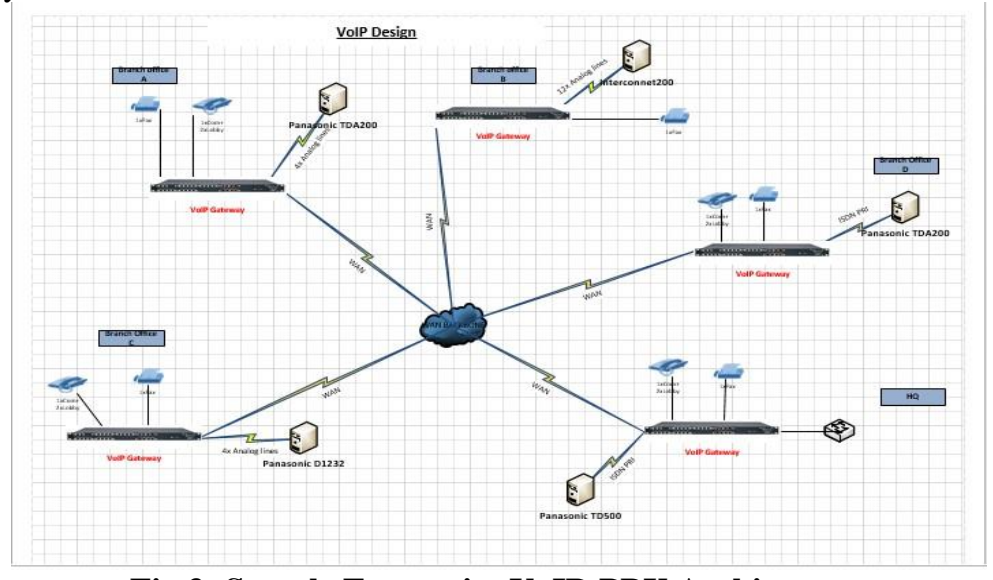

Fig.3: Sample Enterprise VoIP-PBX Architecture 


\subsection{Basic Infrastructure}

Some of the basic elements that are required for Enterprise VoIP-PBX converged implementation are highlighted below. The existing PBX is Integrated using ISDN PRI card to offer smooth calls services transitions.

VoIP Gateway: The media gateway interfaces between the VoIP server and PBX/PSTN. Digital cards like E1 or T1 and analogue are installed on the device for connection to PSTN or PBX devices. Most enterprise VoIP gateway like Audiocodes host both the VoIP server and the gateway on the same appliance.

E1 Interface: E1 is a digital carrier signal that transmit DS-1 signal. E1 is the European term for the transmission (digital signal). The data rate of E1 is about 2 megabits per second. It has 32 channels at the speed of $64 \mathrm{Kbps}$ DS0 signals multiplexed into a single DS1 E1 carrier. Two channels among the 32 are reserved. One channel is used for signaling while the other channel is used for controlling. Framing and Synchronization is at $64 \mathrm{Kbps}$. E1 is used to transmit data and voice signal between devices. The E1 interface card is needed for the PSTN breakout, this will enable the VoIP clients to make and receive calls to and fro PSTN lines like GSM service providers.

ISDN PRI: ISDN (Integrated Services Digital Network) is a digital telephone standard designed to replace analogue connections by utilizing copper wires, fiber optic or microwave to transmit signals from the customer's end to the service providers' premises. The PRI is meant for VoIP server and media gateway integration with the legacy analogue PBX.

WAN Link: Wide Area switching and routing networks are required for moving the VoIP packets across the geographical locations. This WAN link is going to be riding on the CORE network of ISP. Effort should be made to ensure the service provided by the ISPs are reliable enough to avoid packet loss ad jittering.

PABX: Private automatic branch exchange (PABX) is an automatic telephone switching system within a private enterprise organisation. Such systems are also called private branch exchanges (PBX). PABX performs all the switching necessary for making internal calls between extensions within the organizations and external calls between extensions and PSTN lines.

IP Phones: For end users SIP supported phones can be used for making and receiving calls, other alternative is softphone applications that can be installed on laptops. The phones can be programmed with Direct Inward Dial (DID) or normal extension numbers. Most of the IP phones are powered by PoE switches which makes the deployment a lot easier.

\section{SECURING VOIP}

Securing VoIP has many challenges that do not exist in the public switched telephone network (PSTN), a circuit switched system. VoIP as an application running over the internet connection inherits the internet's security issues. This section of the paper identifies the common security issues found in a corporate VoIP implementation and discuss the method use to combat them.

Problem \# 1: VoIP traffic might be internet bound.

Internet is inherently less secure than placing a call over traditional circuit switched networks. The internet is a dangerous place, and packet sniffers can grab unencrypted traffic. Some solution to this is to implement VPN tunnels between the headquarter and branch offices. This will introduce additional payload and headers to voice packets and may result in delayed VoIP packets.

\section{Problem \# 2: Perimeter security options for VoIP are limited.}

Securing VoIP traffic at the firewall level presents certain challenges. First, not all firewalls are VoIP aware. An older firewall may not recognize VoIP protocols such as SIP, MGCP or Cisco's SCCP protocol, and incorrectly block this traffic. It will be necessary to ensure that modern day firewall like Checkpoint and Palo Alto are implemented at the perimeter to prevent intrusion.

\section{Problem \# 3: Denial of Service (DoS)}

Many VoIP implementations may leave TCP/UDP ports unnecessarily open and without sufficient monitoring. These may be seen as potential vulnerability for DoS attack. DoS attack is a concerted, coordinated effort to flood a network node with requests. These attacks can "busy" a system, rendering it unusable for other network services. Best practice to combat these attacks, is to ensure that unnecessary ports and services are shut down or disable, and that the network is properly patched for newly discovered vulnerabilities.

\section{Problem \# 4: Eavesdropping on calls}

Eavesdropping on calls is a major issue with VoIP, many software applications are out there that operates on the network by grabbing packets, which can convert to a wave voice file for listening. The best method of preventing this attack to properly secure access to the call management system and implement encryption on voice traffic. 


\section{QoS IMPLEMENTATION}

Due to the real time nature of voice, congestion can severely affect the quality of VoIP traffic causing poor user experience for the subscribers. In deploying VoIP network, the quality of service (QoS) must be put into consideration during design and as essential component in the operation of the VoIP. It is recommended to take a layered QoS approach which makes it easier to implement and manage QoS policy from end to end of the voice network.

The VoIP's QoS policy should cover Layer 2, Layer 3 and the application layer of the network. This will help assure that the VoIP traffic is given preferential treatment as it is transported from one endpoint to another.

\section{CONCLUSION}

The VoIP technology has been predicted to be the future to Telecommunications globally. This paper presented a methodology on how enterprise VoIP-PBX convergence solution can be deployed successfully in organizations. The methodology can help organizations decide quickly and easily how well to deploy VoIP without having to discard their legacy PBX system.

\section{REFERENCE}

[1].“Critical steps for successful VoIP deployment”, VoIP-WP101-R, April 12, 2005

[2].Dorgham Sisalem, Jiri Kuthan "Understanding SIP",

[3].Wikipedia, $\quad$ complete, $\quad$ sophisticated protocol stack", https://en.wikipedia.org/wiki/H.323\#/media/File:Typical_H.323_Stack.png

[4].Sanjay Kumar Sonkar, Rahul Singh, Ritu Chauhan, Ajay Pal Singh "A Review Paper: Security on Voice over Internet Protocol from Spoofing attacks”, IJARCCE Vol. 1, Issue 3, May 2012, pp. 153-160.

[5].Karapantazis, S. \& Pavlidou, F. (2009) 'VoIP: A comprehensive survey on promising technology', Computer Networks, 53(12), pp.2050-2090

[6].Osanaiye Opeyemi Ayokunle "Integrating Voice over Internet Protocol (VoIP) Technology as a Communication Tool on a Converged Network in Nigeria", Volume 2 No. 11, November 2012,pp 829837.

[7].Matthew Ruck, "Top Ten Security Issues Voice over IP (VoIP)", Project VoIP Security Revision 1.0 Design Data 2010 White Paper Series

[8].A.A. Ojugo, R.E. Yoro, A.O, Eboka., M.O. Yerokun and I.J.B Iyawah "Implementation Issues of VoIP to Enhance Rural Telephony in Nigeria" Journal of Emerging Trends in Computing and Information Sciences Vol. 4, No. 2 Feb 2013, pp 172-179 